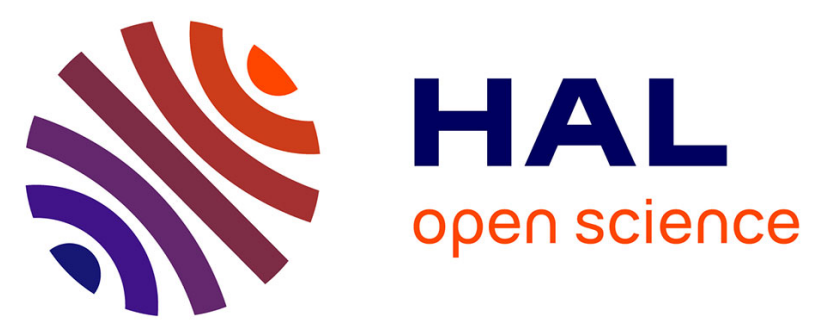

\title{
Contribution to the study of F-G-K-M binaries. X. HD 54901, HD 120544 and HD 123280, three nearby F-type spectroscopic binaries
}

\author{
Jean-Michel Carquillat, Jean-Louis Prieur, Stephane Udry
}

\section{To cite this version:}

Jean-Michel Carquillat, Jean-Louis Prieur, Stephane Udry. Contribution to the study of F-G-K-M binaries. X. HD 54901, HD 120544 and HD 123280, three nearby F-type spectroscopic binaries. Astronomical Notes / Astronomische Nachrichten, 2005, 326 (1), pp.31-37. 10.1002/asna.200410260 . hal-02111216

\author{
HAL Id: hal-02111216 \\ https://hal.science/hal-02111216
}

Submitted on 27 Apr 2019

HAL is a multi-disciplinary open access archive for the deposit and dissemination of scientific research documents, whether they are published or not. The documents may come from teaching and research institutions in France or abroad, or from public or private research centers.
L'archive ouverte pluridisciplinaire HAL, est destinée au dépôt et à la diffusion de documents scientifiques de niveau recherche, publiés ou non, émanant des établissements d'enseignement et de recherche français ou étrangers, des laboratoires publics ou privés. 


\title{
Contribution to the study of F-G-K-M binaries. X. HD 54901, HD 120544 and HD 123280, three nearby F-type spectro- scopic binaries.
}

\author{
J.-M. Carquillat ${ }^{1}$, J.-L. Prieur ${ }^{1}$, and S. Udry ${ }^{2}$ \\ 1 UMR 5572 d'Astrophysique, Observatoire Midi-Pyrénées - CNRS, 14, Avenue Edouard Belin, 31400 Toulouse, France. \\ 2 Observatoire de Genève, 51 chemin des Maillettes, CH-1290 Sauverny, Switzerland.
}

\author{
Received April 27, 2019; accepted
}

\begin{abstract}
The orbital elements of HD 54901, HD 120544 and HD 123280, three nearby F-type spectroscopic binaries, are presented. They are based on observations made between 1982 and 2004 with the CORAVEL instrument of Observatoire de Haute-Provence. Physical parameters are derived for the two components of HD 54901 (SB2) and for the primaries of HD 120544 and HD 123280. The rotation-revolution synchronism of the detected components is investigated. Pseudosynchronism is very likely achieved by the F7 V secondary component of HD 54901, whereas the F2/3 IV primary has not yet reached this stage.
\end{abstract}

(C) 0000 WILEY-VCH Verlag GmbH \& Co. KGaA, Weinheim

\section{Introduction}

This paper is the tenth of a series (Nadal et al., 1983, Paper I) devoted to the study of late-type stars suspected to be spectroscopic binaries (SB) in the literature, in order to determine their orbital elements. Indeed, when we started this observing program, the statistics showed a lack of SBs with known orbits among the stars of the later spectral types (Carquillat, Ginestet \& Pédoussaut 1971). Most of the observations presented here were obtained with the CORAVEL instrument of the Observatoire de HauteProvence (OHP). HD 54901 and HD 123280 were previously reported as spectroscopic binaries in the General Catalogue of Stellar Radial Velocities (Wilson 1953), the main source of objects for this series. The binary nature of HD 120544 was suspected from the composite spectral classification (G5 III-IV: + A5:) given by Cowley (1973). While this study was still in progress, a preliminary orbit was published by Nordström et al. (1997) for HD 54901, in the context of an investigation of the radial velocities, rotations and duplicity of a large sample of F-type dwarf stars. For that star we provide here an improved orbit based both on our observations and on those of Nordström et al., whereas we give the first (to our knowledge) spectroscopic orbits of HD $\mathbf{1 2 0 5 4 4}$ and HD 123280. Note that HD 54901 is a two-lined spectroscopic binary (SB2), while the two other stars are single-lined binaries (SB1).

\section{Observations and derivation of the orbital elements}

Most of the observations were made with the CORAVEL spectrometer mounted at the Cassegrain focus of the 1-m

The \correspondence command is obsolete!
Swiss telescope during the period 1982-1998, with some complementary observations of HD 120544 after this date (until 2004). This instrument allows measurements of heliocentric radial velocities (RV) by performing a crosscorrelation of the stellar spectrum with a physical mask placed in the focal plane of the spectrograph (Baranne, Mayor \& Poncet 1979). The RVs thus obtained were reduced to the RV data base system of the Geneva Observatory (Udry, Mayor \& Queloz 1999). The orbital elements were determined with our programs "BS1" and "BS2" specially designed for SB1 and SB2 systems. They are based on an iterative scheme of Gauss-Newton type that performs a least-squares minimization of the residuals, starting from an initial guess of the orbital parameters.

For HD 54901 we used 50 observations with CORAVEL and 22 others from Nordström et al. (1997) (Table 1). Weights of 1 were assigned to the RVs of the primary component, and weights of 0.35 to those of the secondary according to their mean standard errors.

The orbital elements of HD 120544 were computed from 32 CORAVEL RVs ( 30 at OHP and 2 with the ESO CORAVEL at La Silla, by Andersen et al. (1985)) plus 4 RVs obtained from photographic spectra with IIa O plates) in 1985-1989 with the spectrograph mounted at the coudé focus of the OHP $1.5-\mathrm{m}$ telescope (Table 2). The RVs measured from the photographic spectra, initially obtained with Fehrenbach (1972)'s tables, were corrected by an offset of $+2.9 \mathrm{~km} \cdot \mathrm{s}^{-1}$ to make them consistent with CORAVEL measurements. They were assigned a 0.3 weight (whereas a weight of 1 was used for CORAVEL velocities), according to their mean standard error.

For HD 123280, all the $48 \mathrm{RVs}$ of Table 3 derive from CORAVEL (OHP) observations.

The orbital elements we found are given in Table 4, and the corresponding radial velocity curves are shown in 
Fig. 1. For HD 54901, the orbit we obtain confirms and improves the orbit of Nordström et al. (1997). In all cases, the standard deviation of the residuals, $\sigma_{(O-C)}$, is smaller than the mean internal error of the RVs, which indicates the absence of detectable spectroscopic third bodies in those systems.

\section{Physical parameters}

\subsection{General procedure}

In this section, we estimate some physical parameters (Table 5) from the complementary data available for those stars, mainly the parallaxes, $V$ and $B-V$ magnitudes, and Strömgren photometry. Those three stars have parallaxes quoted in the Hipparcos catalogue (ESA 1997), which are given in Col. 4, with the corresponding errors. Those values lead to distances between 90 and $102 \mathrm{pc}$, which are small enough for the interstellar absorption effects to be neglected. The corresponding visual absolute magnitudes are given in Col. 5. Strömgren photometry measurements are also available for those stars (Hauck \& Mermilliod 1998). We used the grid of Moon \& Dworetsky (1985), $c_{0}$ versus $\beta$, to estimate both the effective temperature, $T_{\text {eff }}(\mathrm{Col} .6)$, and the surface gravity, $\log g$ (Col. 7), of the primary components. The metallicities, $[\mathrm{Fe} / \mathrm{H}](\mathbf{C o l}$. 8) were obtained using Cayrel's relation (Crawford 1975). For the two stars for which $H \beta$ photometry was not available (HD 54901 and HD 123280), we estimated the $\beta$ parameter from the $b-y$ value via the correlation relation between those two quantities as given by Crawford (1975). Finally, we report (Fig.2) the positions of the components of those systems (primaries only for HD 120544 and HD 123280, the two components for HD 54901) in the HR theoretical diagram, $\log \left(L / L_{\odot}\right)$ versus $\log T_{\text {eff }}$, computed by Schaller et al. (1992) for stars of solar metallicity and completed with the isochrones computed by Meynet, Mermilliod \& Maeder (1993). The absolute bolometric magnitudes required to obtain $L / L_{\odot}$ were calculated from the values of $M_{v}$, applying the bolometric corrections tabulated by Flower (1996) for the appropriate temperatures. The positions of the stars in the HR diagram (Fig.2) lead to theoretical estimates of their masses, radius and ages, shown in Cols. 9, 10 and 11 of Table 5, respectively.

\subsection{Discussion of individual cases}

\subsubsection{HD 54901}

For this SB2, there exist some observational constraints which allow us to propose a coherent model for the system. These constraints are as follows:

(1) The magnitude difference as deduced from the ratio of the correlation dip areas given by CORAVEL. Indeed we have the relation: $\Delta m_{v}=2.5 \log \left[\left(W_{1} / W_{2}\right) \times\right.$ $\left.\left(T_{\text {eff2 }} / T_{\text {eff1 }}\right)\right]$, where $W$ is the equivalent width, in

Table 1 Radial velocities and $(O-C)$ residuals for HD 54901. Measurements from Nordström et al. (1997) are marked with ${ }^{N}$

\begin{tabular}{|c|c|c|c|c|c|}
\hline $\begin{array}{c}\text { Date (JD) } \\
2400000+\end{array}$ & Cycle & $\begin{array}{r}R V_{1} \\
\mathrm{~km} \cdot \mathrm{s}^{-1}\end{array}$ & $\begin{array}{c}(O-C)_{1} \\
\mathrm{~km} \cdot \mathrm{s}^{-1}\end{array}$ & $\begin{array}{r}R V_{2} \\
\mathrm{~km} \cdot \mathrm{s}^{-1}\end{array}$ & $\begin{array}{r}(O-C)_{2} \\
\mathrm{~km} . \mathrm{s}^{-1}\end{array}$ \\
\hline 46335.68 & -33.50 & -27.6 & -1.5 & 11.9 & 0.3 \\
\hline 46721.69 & -24.08 & 0.8 & 0.4 & -22.7 & 0.6 \\
\hline 46865.36 & -20.58 & -24.1 & 0.3 & 8.0 & -1.3 \\
\hline 47098.69 & -14.89 & 6.4 & 0.7 & -32.8 & -2.7 \\
\hline 47216.58 & -12.01 & $55.1^{N}$ & 0.7 & $-93.7^{N}$ & 0.3 \\
\hline 47461.65 & -6.04 & 32.0 & 0.4 & - & - \\
\hline 47462.93 & -6.00 & $59.7^{N}$ & -0.3 & $-99.9^{N}$ & 1.4 \\
\hline 47464.97 & -5.95 & $36.4^{N}$ & -1.7 & $-73.5^{N}$ & -0.8 \\
\hline 47484.75 & -5.47 & $-25.8^{N}$ & 0.7 & $10.1^{N}$ & -1.9 \\
\hline 47511.66 & -4.82 & $-9.7^{N}$ & -0.9 & $-9.4^{N}$ & 1.7 \\
\hline 47542.63 & -4.06 & $10.8^{N}$ & -0.8 & $-39.4^{N}$ & -1.5 \\
\hline 47570.62 & -3.38 & $-27.3^{N}$ & -0.4 & $11.0^{N}$ & -1.5 \\
\hline 47599.52 & -2.67 & $-19.9^{N}$ & 0.9 & $7.0^{N}$ & 2.5 \\
\hline 47600.35 & -2.65 & -20.8 & 0.9 & - & - \\
\hline 47607.41 & -2.48 & -28.0 & -1.6 & - & - \\
\hline 47640.52 & -1.67 & $-20.5^{N}$ & 0.3 & $6.5^{N}$ & 1.9 \\
\hline 47804.87 & 2.34 & $-19.9^{N}$ & 1.3 & $5.5^{N}$ & 0.4 \\
\hline 47813.81 & 2.55 & $-26.5^{N}$ & 0.2 & $9.8^{N}$ & -2.5 \\
\hline 47821.91 & 2.75 & $-23.7^{N}$ & 0.7 & $8.1^{N}$ & -1.1 \\
\hline 47830.84 & 2.97 & $34.8^{N}$ & -0.6 & $-71.1^{N}$ & -2.0 \\
\hline 47865.79 & 3.82 & $-20.3^{N}$ & -0.5 & $3.3^{N}$ & 0.0 \\
\hline 47867.64 & 3.87 & -13.8 & 0.1 & -4.8 & -0.3 \\
\hline 47870.68 & 3.94 & 10.4 & -1.6 & -38.5 & -0.1 \\
\hline 47871.58 & 3.96 & 29.0 & -0.3 & - & - \\
\hline 47872.58 & 3.99 & 54.3 & 0.9 & - & - \\
\hline 47874.56 & 4.04 & 47.0 & 0.7 & -84.1 & -0.8 \\
\hline 47896.84 & 4.58 & $-26.8^{N}$ & 0.1 & $13.1^{N}$ & 0.6 \\
\hline 47923.72 & 5.23 & $-13.8^{N}$ & 0.6 & - & - \\
\hline 47955.59 & 6.01 & $61.8^{N}$ & 0.4 & $-103.6^{N}$ & -0.4 \\
\hline 47964.35 & 6.23 & -13.7 & -0.1 & -4.5 & 0.4 \\
\hline 47965.37 & 6.25 & -17.4 & -1.6 & -1.0 & 1.0 \\
\hline 47966.45 & 6.28 & -18.5 & -0.7 & 1.3 & 0.7 \\
\hline 47967.39 & 6.30 & -18.3 & 1.0 & 3.4 & 0.9 \\
\hline 47969.43 & 6.35 & -21.7 & 0.1 & 5.5 & -0.4 \\
\hline 47987.52 & 6.79 & $-22.0^{N}$ & 0.3 & $8.3^{N}$ & 1.8 \\
\hline 48167.91 & 11.19 & $-10.4^{N}$ & -0.9 & $-9.5^{N}$ & 0.7 \\
\hline 48194.88 & 11.85 & $-16.3^{N}$ & 0.5 & $0.6^{N}$ & 1.3 \\
\hline 48261.55 & 13.47 & -27.7 & -2.1 & 9.8 & -1.1 \\
\hline 48262.43 & 13.49 & -26.4 & -0.4 & 10.7 & -0.7 \\
\hline 48265.58 & 13.57 & -27.5 & -0.7 & - & - \\
\hline 48291.67 & 14.21 & $-11.1^{N}$ & 0.7 & $-9.5^{N}$ & -2.2 \\
\hline 48609.80 & 21.97 & $33.2^{N}$ & -0.1 & $-65.1^{N}$ & 1.3 \\
\hline 48671.44 & 23.47 & -25.8 & -0.3 & 9.0 & -1.8 \\
\hline 48676.41 & 23.59 & -27.0 & -0.1 & 12.9 & 0.3 \\
\hline 48937.64 & 29.96 & 30.1 & 1.2 & -62.4 & -1.9 \\
\hline 48938.68 & 29.99 & 53.5 & -0.4 & -92.8 & 0.5 \\
\hline 48940.55 & 30.03 & 48.6 & 0.6 & -83.7 & 1.9 \\
\hline 48967.59 & 30.69 & -26.1 & 0.0 & 11.6 & 0.1 \\
\hline 48969.57 & 30.74 & -23.6 & 1.2 & 10.5 & 0.7 \\
\hline 49325.52 & 39.42 & -24.7 & -0.3 & 8.8 & -0.5 \\
\hline 49427.37 & 41.91 & -3.9 & 0.3 & -17.3 & -0.1 \\
\hline 49428.34 & 41.93 & 6.3 & 0.6 & -30.1 & 0.1 \\
\hline 49429.36 & 41.95 & 22.2 & 0.1 & -49.1 & 2.6 \\
\hline 49430.34 & 41.98 & 44.4 & -0.7 & -82.9 & -1.1 \\
\hline 49431.35 & 42.00 & 61.8 & -0.4 & -104.6 & -0.4 \\
\hline 49432.33 & 42.03 & 52.1 & -0.5 & -93.4 & -1.8 \\
\hline 49432.46 & 42.03 & 49.4 & -0.8 & -89.1 & -0.7 \\
\hline 49640.71 & 47.11 & 7.3 & 0.5 & -33.6 & -2.0 \\
\hline 49781.41 & 50.54 & -26.0 & 0.6 & - & - \\
\hline 49783.41 & 50.59 & -25.2 & 1.7 & 14.0 & 1.5 \\
\hline 49784.43 & 50.61 & -26.5 & 0.4 & 13.2 & 0.6 \\
\hline 49786.37 & 50.66 & -25.7 & 0.9 & 14.4 & 2.3 \\
\hline 50125.49 & 58.93 & 6.3 & -0.7 & -34.3 & -2.4 \\
\hline 50127.47 & 58.98 & 47.1 & -0.1 & -84.3 & 0.3 \\
\hline 50325.66 & 63.81 & -21.6 & -1.1 & 2.9 & -1.2 \\
\hline 50415.62 & 66.01 & 63.3 & 1.1 & -101.9 & 2.3 \\
\hline 50418.62 & 66.08 & 18.7 & 1.4 & -43.5 & 1.8 \\
\hline 50419.60 & 66.11 & 9.0 & 0.9 & -30.5 & 2.8 \\
\hline 50420.66 & 66.13 & 0.0 & -0.9 & -25.3 & -1.5 \\
\hline 50477.45 & 67.52 & - & - & 12.5 & 0.7 \\
\hline 50479.50 & 67.57 & - & - & 11.9 & -0.5 \\
\hline 50745.65 & 74.06 & 29.8 & -0.8 & -62.8 & 0.0 \\
\hline
\end{tabular}



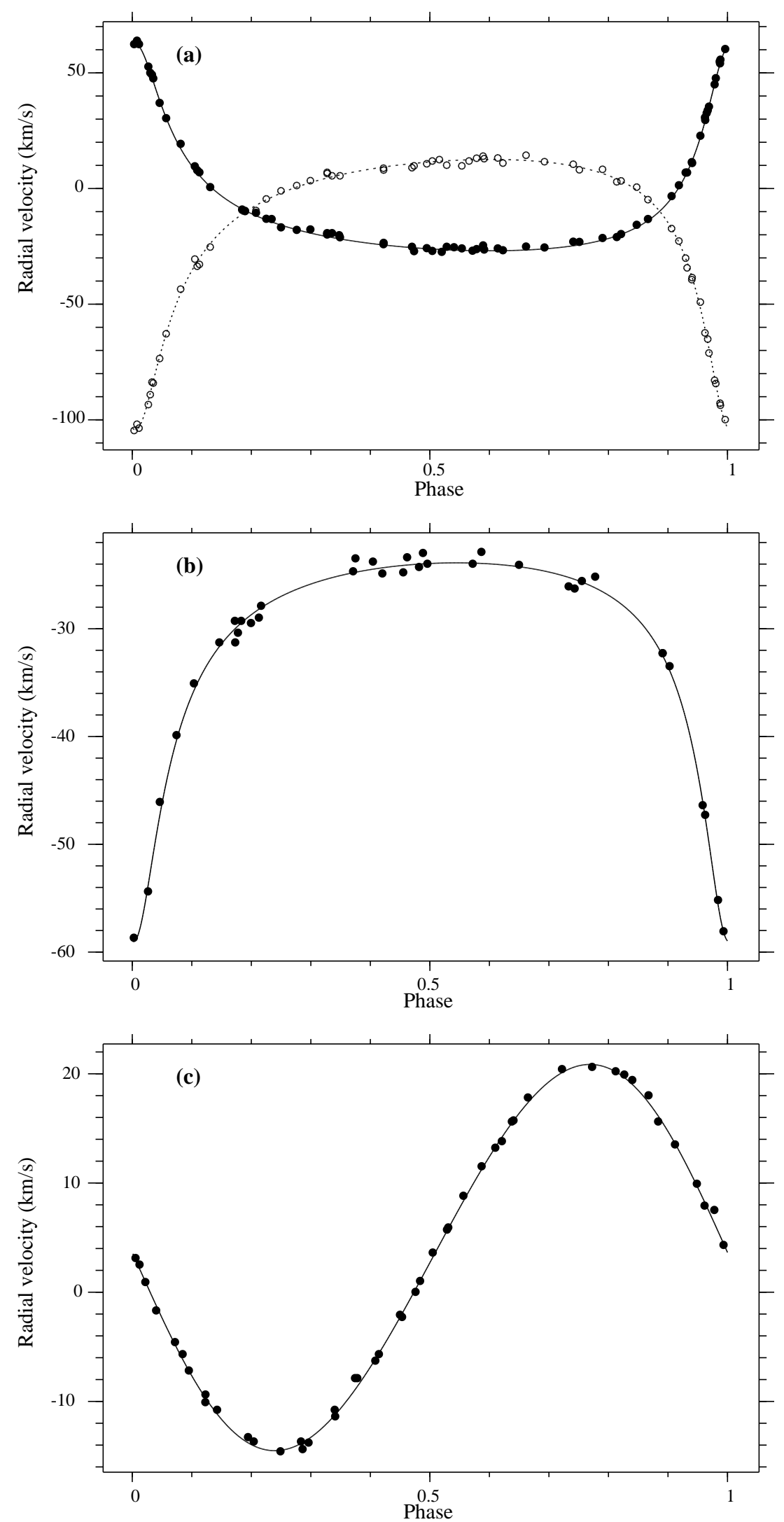

Fig. 1 RV curves computed with the orbital elements of Tables 4: (a) HD 54901, (b) HD 120544, and (c) HD 123280. The origin of the phases corresponds to the periastron passage. 
Table 2 Radial velocities and $(O-C)$ residuals for HD 120544 Photographic measurements are marked with ${ }^{P}$ and measurements from Andersen et al. (1985) with ${ }^{A}$.

\begin{tabular}{|c|c|c|c|}
\hline $\begin{array}{l}\text { Date (JD) } \\
2400000+\end{array}$ & Cycle & $\begin{array}{r}R V \\
\mathrm{~km} \cdot \mathrm{s}^{-1}\end{array}$ & $\begin{array}{c}(O-C) \\
\mathrm{km}_{\mathrm{sm}} \mathrm{s}^{-1}\end{array}$ \\
\hline 44645.83 & -4.26 & $-26.4^{A}$ & -1.0 \\
\hline 44976.75 & -0.54 & -24.9 & -0.8 \\
\hline 44979.70 & -0.51 & -23.1 & 0.9 \\
\hline 45127.50 & 1.15 & $-31.4^{A}$ & 0.3 \\
\hline 45343.74 & 3.57 & -24.1 & -0.2 \\
\hline 46174.52 & 12.89 & $-32.4^{P}$ & 0.1 \\
\hline 46225.39 & 13.46 & -23.5 & 0.6 \\
\hline 46823.68 & 20.17 & $-31.4^{P}$ & -1.4 \\
\hline 46901.54 & 21.05 & $-46.2^{P}$ & 1.1 \\
\hline 47565.64 & 28.50 & $-24.1^{P}$ & -0.1 \\
\hline 47966.59 & 32.99 & -58.2 & -0.1 \\
\hline 47969.55 & 33.03 & -54.5 & -0.6 \\
\hline 48670.64 & 40.89 & -32.4 & 0.1 \\
\hline 48671.69 & 40.90 & -33.6 & 0.3 \\
\hline 48676.64 & 40.96 & -46.5 & -0.5 \\
\hline 49142.42 & 46.18 & -29.4 & 0.1 \\
\hline 49145.39 & 46.22 & -28.0 & 0.1 \\
\hline 49426.62 & 49.37 & -24.8 & 0.0 \\
\hline 49429.59 & 49.40 & -23.9 & 0.6 \\
\hline 49783.56 & 53.37 & -23.6 & 1.2 \\
\hline 49787.59 & 53.42 & -25.0 & -0.7 \\
\hline 50125.69 & 57.21 & -29.1 & -0.9 \\
\hline 50192.53 & 57.96 & -47.4 & 0.1 \\
\hline 50194.46 & 57.98 & -55.3 & 0.3 \\
\hline 50478.68 & 61.17 & -29.4 & 0.6 \\
\hline 50610.39 & 62.65 & -24.2 & 0.0 \\
\hline 50835.73 & 65.18 & -30.5 & -0.8 \\
\hline 50837.69 & 65.20 & -29.6 & -0.9 \\
\hline 50974.41 & 66.73 & -26.2 & -1.0 \\
\hline 50976.39 & 66.76 & -25.7 & 0.0 \\
\hline 50978.39 & 66.78 & -25.3 & 0.9 \\
\hline 51185.73 & 69.10 & -35.2 & 0.5 \\
\hline 52781.39 & 87.00 & -58.8 & 0.2 \\
\hline 53011.74 & 89.59 & -23.0 & 0.9 \\
\hline 53091.55 & 90.48 & -24.4 & -0.4 \\
\hline 53144.37 & 91.07 & -40.0 & 0.4 \\
\hline
\end{tabular}

$\mathrm{km} . \mathrm{s}^{-1}$, of the correlation dip, and $T_{\text {eff }}$ the effective temperature of the component, the subscripts 1 , 2 referring to the primary and the secondary, respectively (Duquennoy 1994, private communication). For HD 54901, we have $W_{1} / W_{2}=3.48$, that leads to $\Delta m_{v}=1.35$, as a first approximation assuming $T_{\text {eff1 }} \sim$ $T_{\text {eff2 }}$.

(2) The global visual absolute magnitude $M_{v}=2.3$.

(3) The global $B-V$ colour index, $B-V=0.40$.

(4) The mean temperature from the Strömgren photometry, $T_{\text {eff }}=6800 \mathrm{~K}$.

(5) The mass-ratio $K_{1} / K_{2}=0.76$ (Cf. Table 4).

Taking into account all those constraints, and proceeding by an iterative method, we finally obtained for the system the classification (F2/3 IV + F7 V), with $\Delta m_{v}=1.25$; $T_{\text {eff1 }}=6940 \mathrm{~K} ; T_{\text {eff } 2}=6320 \mathrm{~K} ; M_{v 1} \approx 2.6 ; M_{v 2} \approx 3.9$. The theoretical masses: $M_{1} \approx 1.55 M_{\odot}, M_{2} \approx 1.20 M \odot$ (Fig. 2) are in very good agreement with the mass-ratio deduced from the orbit. This is also close to the classification of F2 III for this system given by Nassau \& MacRae (1955) from objective-prism observations.
Table 3 Radial velocities and $(O-C)$ residuals for HD 123280.

\begin{tabular}{rrrr}
\hline Date $(\mathrm{JD})$ & Cycle & $\begin{array}{r}R V \\
2400000+\end{array}$ & $\begin{array}{r}(O-C) \\
\mathrm{km} . \mathrm{s}^{-1}\end{array}$ \\
\hline 44979.75 & -0.99 & 3.0 & 0.0 \\
45345.72 & 9.07 & -4.7 & 0.1 \\
46226.42 & 33.30 & -13.9 & -0.5 \\
46229.39 & 33.38 & -8.0 & 0.6 \\
46230.50 & 33.41 & -6.4 & -0.3 \\
46486.62 & 40.45 & -2.4 & -0.4 \\
47287.55 & 62.48 & 0.9 & -0.1 \\
47601.64 & 71.12 & -10.2 & -0.5 \\
47604.59 & 71.20 & -13.8 & 0.3 \\
47607.48 & 71.28 & -13.8 & 0.1 \\
47609.54 & 71.34 & -10.9 & 0.4 \\
48670.72 & 100.53 & 5.6 & 0.0 \\
48671.72 & 100.56 & 8.7 & 0.4 \\
48673.67 & 100.61 & 13.1 & -0.2 \\
48674.68 & 100.64 & 15.5 & 0.0 \\
48675.66 & 100.66 & 17.7 & 0.3 \\
49140.47 & 113.45 & -2.2 & 0.1 \\
49141.42 & 113.48 & -0.1 & -0.4 \\
49142.47 & 113.50 & 3.5 & 0.3 \\
49143.42 & 113.53 & 5.8 & 0.0 \\
49145.46 & 113.59 & 11.4 & 0.2 \\
49147.41 & 113.64 & 15.6 & -0.1 \\
49428.57 & 121.37 & -8.0 & 0.9 \\
49781.60 & 131.08 & -5.8 & 0.3 \\
49783.71 & 131.14 & -10.9 & 0.2 \\
49785.60 & 131.19 & -13.4 & 0.4 \\
49787.58 & 131.25 & -14.7 & -0.2 \\
49808.58 & 131.83 & 19.8 & 0.1 \\
49813.48 & 131.96 & 7.8 & -0.5 \\
50164.66 & 141.62 & 13.7 & -0.5 \\
50171.61 & 141.81 & 20.1 & -0.1 \\
50172.62 & 141.84 & 19.3 & 0.3 \\
50173.62 & 141.87 & 17.9 & 0.5 \\
50176.57 & 141.95 & 9.8 & 0.0 \\
50177.64 & 141.98 & 7.4 & 1.1 \\
50193.50 & 142.41 & -5.8 & -0.2 \\
50324.31 & 146.01 & 2.4 & 0.2 \\
50325.32 & 146.04 & -1.8 & -0.6 \\
50327.32 & 146.10 & -7.3 & -0.1 \\
50328.34 & 146.12 & -9.5 & 0.2 \\
50479.69 & 150.29 & -14.5 & -0.7 \\
50481.68 & 150.34 & -11.5 & -0.3 \\
50610.48 & 153.88 & 15.5 & -0.6 \\
50611.50 & 153.91 & 13.4 & -0.2 \\
50614.47 & 153.99 & 4.2 & -0.3 \\
50615.51 & 154.02 & 0.8 & -0.2 \\
50822.74 & 159.72 & 20.3 & 0.2 \\
50824.58 & 159.77 & 20.5 & -0.4 \\
\hline & & &
\end{tabular}

\subsubsection{HD 120544}

Several spectral classifications in the MK system have been given for that star. In Sect. 1, we have already mentioned the composite spectral classification (G5 III-IV: + A5:) given by A. Cowley (1973). However, this first classification was never confirmed by subsequent studies. Other classifiers did not recognize a composite spectrum for HD 120544: they only mention the spectrum of an $F$ star alone. Malaroda (1975) gives F7 IV, A.P. Cowley (1976) gives F7 V, and Houk \& Smith-Moore (1988) give F6 IV/V. To investigate this problem, we included this star in our observational program of classification of stars with composite spectra in the near infrared (Ginestet et al. 1997). Indeed we reached the same conclusion and proposed, tentatively, the spectral type F5 V. Hence the classification proposed by A. Cowley is very probably erroneous.

With its low mass-function of $0.02 \mathrm{M}_{\odot}$, the undetected secondary component of HD 120544 is likely to be faint with a low mass. Hence we have neglected its contribution 
to the magnitude and photometric indices of the star, and determined the location of the primary component in the HR diagram (Fig. 2). The comparison with theoretical evolution tracks suggests this object is an already evolved star, not a dwarf, near the turn-off point. The visual absolute magnitude, close to 1.8 , is consistent with the type F6 III-IV (Schmidt-Kaler 1982). Note nevertheless that the luminosity class is difficult to estimate with accuracy, from visual classifications, in the range III-V for the F stars.

\subsubsection{HD 123280}

The SIMBAD data base refers to only one MK spectral classification for this star, F6 V, given by Moore \& Paddock (1950) from observations at Lick Observatory. Those observations also permitted to reveal the binary nature of HD 123280. As for HD 120544, the low mass-function of $0.02 \mathrm{M}_{\odot}$ suggests a SB1 system with a faint companion, and the contribution of the secondary can be neglected. In this case, the physical parameters we find and the position in the HR diagram suggest that the primary is a bona fide dwarf star and confirm the validity of the classification F6 V.

\section{Separations and minimum masses of the unseen companions}

\subsection{HD 54901}

For this $\mathrm{SB} 2$, we know $a \sin i=a_{1} \sin i+a_{2} \sin i=$ $45.20 \mathrm{Gm}$. An estimation of $i$ can be deduced from the quantity $M_{1} \sin ^{3} i$ and the theoretical mass $M_{1}=1.55 \mathrm{M}_{\odot}$, i.e. $\sin i=0.930$, or $i=68^{\circ}$. We thus obtain $a=$ $48.60 \mathrm{Gm}=0.32$ a.u., which corresponds to a mean angular separation of 3.4 mas taking into account the parallax of the system. This separation makes this system resolvable with multi-pupil large interferometers.

\subsection{HD 120544 and HD 123280}

Writing the mass-function: $f(m)=M_{1} \times \sin ^{3} i \times \mu^{3} /(1+$ $\mu)^{2}$, where $\mu=M_{2} / M_{1}$ is the mass-ratio, we calculated for each system the minimum mass of the secondary, $M_{2 \mathrm{~min}}$, assuming for the primary the theoretical mass $M_{1}$ (Table 5, Col. 9) from the position of the star in the HR diagram. We found $M_{2 \min }=0.5 \mathrm{M}_{\odot}$ and $0.4 \mathrm{M}_{\odot}$ for HD 120544 and HD 123280, respectively. These masses are those of cool red dwarf stars.

For a given value of the orbital inclination $i$, the mean linear separation $a$ can be computed from the following relation: $a=a_{1}+a_{2}=a_{1} \sin i \times(1+1 / \mu) / \sin i$, where $a_{1} \sin i$ can be estimated from the orbit (Table 4, Col. 8). We have shown (Carquillat at al. 1982) that the values of $a$ obtained using this relation are not very sensitive to the value of $i$, even when $i$ varies over a large range of orbital inclinations. Hence, we calculated $a$ for the two systems using the statistical most likely value of $i=60^{\circ}$. For HD 120544 and HD 123280, we found $a=76.71 \mathrm{Gm}=0.51$ a.u., and $a=38.80 \mathrm{Gm}=0.26$ a.u., respectively. This corresponds to angular separations of 5.7 mas and 2.5 mas, respectively. As for HD 54901, the angular separations lie in the possibilities of optical interferometers, but in this case the low luminosity of the secondaries makes them unlikely to be detected with current available interferometers.

\section{Tests of rotation-revolution synchronism}

\subsection{Introduction of the method}

Tidal interaction between the two components of binary systems can lead either to a spiraling in of the two stars, followed by a collision, or to the evolution towards an equilibrium state characterized by circularity of the orbit, coplanarity and synchronism of the orbital and spin motions. Classically, this rotation-revolution synchronism occurs in a close system with a circular or quasi-circular orbit. Then the spin velocity $\Omega=V_{e} / R$ is equal to the mean angular orbital velocity $\dot{\theta}=2 \pi / P$, i.e.

$$
V_{e} \times P=50.6 \times R,
$$

where $V_{e}$ is the equatorial rotational velocity in $\mathrm{km} . \mathrm{s}^{-1}, P$ the orbital period in days and $R$ the radius of the star, in solar radii.

The time scales for circularization and synchronization are generally different. In detached systems where the orbital angular momentum is much larger than the rotational angular momentum at the equilibrium configuration, Hut (1981) has shown that coplanarity and synchronism of the spin and orbital motions are reached rather quickly, whereas the eccentricity of the orbit diminishes more slowly. Note that Hut's model was the "weak friction model", i.e. considering the equilibrium tide only. Zahn $(1975,1977)$ studied the dynamical tides, where the stars oscillate, and reached the same conclusions. Hence the orbits of binary stars are expected to remain rather eccentric for a time much longer than the time needed for synchronization.

In the case of an eccentric orbit, the tidal interaction is much more efficient near the periastron passage. Indeed, the disturbing acceleration which causes a slow change of the orbital parameters is proportional to $r^{-7}$, where $r$ is the distance between the two components. Hut (1981) proposed that the synchronism is first reached in this part of the orbit only: this is the pseudo-synchronism. He defines the spin velocity of pseudo-synchronism $\Omega_{\mathrm{ps}}$ as the equilibrium (minimum) value for the spin velocity obtained through energy dissipation by tidal effects, considering the present value of the eccentricity $e$ as constant. In reality, the eccentricity decreases slowly with time, and hence $\Omega_{\mathrm{ps}}$ is larger than $\Omega_{\infty}$, the value of the spin velocity when the full equilibrium is reached (with $e=0$ ). When a component rotates with $\Omega=\Omega_{\mathrm{ps}}$, it is qualified as "pseudo-synchronized". The corresponding spin period $P_{\mathrm{ps}}=2 \pi / \Omega_{\mathrm{ps}}$, is called 
pseudo-synchronisation period. For such a system, the instantaneous angular orbital velocity at the periastron is close to (but slightly different from) $\Omega_{\mathrm{ps}}$.

Hut (1981) has found that:

$$
P_{\mathrm{ps}}=P \times \frac{\left(1+3 e^{2}+\frac{3}{8} e^{4}\right)\left(1-e^{2}\right)^{3 / 2}}{\left(1+\frac{15}{2} e^{2}+\frac{45}{8} e^{4}+\frac{5}{16} e^{6}\right)}
$$

An observational test for pseudo-synchronism can be expressed by the condition that the observed angular rotation of the star is equal to $\Omega_{\mathrm{ps}}$ :

$$
\frac{V_{e}}{R}=\frac{2 \pi}{P_{\mathrm{ps}}}
$$

The values of the projected equatorial velocities $V_{e} \sin i$ (generally called $v \sin i$ ), can be deduced from the profiles of the CORAVEL correlation dips, with the method described in Benz \& Mayor $(1981,1984)$. They are given in Table 5 (Col. 3). The values of $V_{e}$ remain unfortunately not accessible since the inclination $i$ is unknown. By multiplying the two members of (3) by $\sin i$ we obtain: $v \sin i=50.6 R \sin i / P_{\mathrm{ps}}$ (with the same units as relation (1)). Hence

$$
v \sin i \leq 50.6 R / P_{\mathrm{ps}}
$$

appears as a condition of pseudo-synchronism (and even of synchronism since $P_{\mathrm{ps}}=P$ when $e=0$ ), when $i$ is unknown.

\subsection{The SB1s: HD 120544 and HD 123280}

For HD 120544, which has a notable eccentricity, the relation (2) gives $P_{\mathrm{ps}}=19.07 \mathrm{~d}$, and (4) gives $v \sin i \leq$ $8.5 \pm 1.0 \mathrm{~km} . \mathrm{s}^{-1}$, which is incompatible with the value of $23.5 \pm 2.4 \mathrm{~km} . \mathrm{s}^{-1}$, quoted in Table 5 .

For HD 123280, which has a quasi-circular orbit, the relation (4) with $P_{\mathrm{ps}} \approx P$ gives $v \sin i \leq 1.9 \pm$ $0.2 \mathrm{~km} . \mathrm{s}^{-1}$, also incompatible with the observed value $\left(8.6 \pm 0.5 \mathrm{~km} . \mathrm{s}^{-1}\right)$. We conclude that the primaries of those two systems rotate too fast for synchronism or pseudosynchronism.

\subsection{The SB2 HD 54901}

Like HD 120544, HD 54901 has a notable eccentricity. The period of pseudo-synchronism is $P_{\mathrm{ps}}=8.89 \mathrm{~d}$. Applying the relation (4) to the primary, we find: $v_{1} \sin i \leq$ $10.3 \pm 1.4 \mathrm{~km} . \mathrm{s}^{-1}$ as a condition of pseudo-synchronism, which is not compatible with the observed value $(16.3 \pm$ $1.0 \mathrm{~km} . \mathrm{s}^{-1}$ ). Applying the same relation to the secondary, we find $v_{2} \sin i \leq 7.1 \pm 0.9 \mathrm{~km} . \mathrm{s}^{-1}$. For this component, the condition (4) appears to be verified by the observed value $\left(8.5 \pm 1.0 \mathrm{~km} . \mathrm{s}^{-1}\right)$. More precisely, if we adopt for $\sin i$ the assumed value of 0.93 (see Sect. 4.1), the pseudo-synchronism hypothesis requires $v \sin i=6.6 \pm$ $1.0 \mathrm{~km} . \mathrm{s}^{-1}$, value which remains compatible with the observed value within the errors. We conclude that the secondary of HD 54901 has probably reached the state of pseudo-synchronism.
This result could be explained by the fact that the secondary, cooler than the primary, should have a thicker convective zone and consequently should be the first component to be synchronized. Indeed, theoretical works (e.g., Zahn, 1975, 1977) have shown that tidal interaction is much more effective in stars possessing a convective envelope, and the main dissipation process is then convective turbulence.

Acknowledgements. This work is based on observations made at the Haute-Provence Observatory (France).

We are indebted to M. Mayor, Director of Geneva Observatory, for giving us observing time with CORAVEL. We thank B. Pernier, J.-L. Halbwachs, M. Imbert, and R. Griffin for making some observations of HD 120544 and HD 123280 at our request. For bibliographic references, we used the SIMBAD data base, operated by the "Centre de Données Astronomiques de Strasbourg" (France).

\section{References}

Andersen, J., Nordström, B., Ardeberg, A., Benz, W., Imbert, M., Lindgren, H., Martin, N., Maurice, E., Mayor, M., Prévot, L.: 1985, A\&AS 59, 15

Baranne, A., Mayor, M., \& Poncet, J.L.: 1979, Vistas Astron., 23, 279

Benz, W., Mayor, M.: 1981, A\&A, 93, 235

Benz, W., Mayor, M.: 1984, A\&A, 138, 183

Carquillat, J.-M., Ginestet, N., Pédoussaut, A.: 1971, Sciences, Tome II-N ${ }^{\circ} 4,251$

Carquillat, J.-M., Nadal, R., Ginestet, N., Pédoussaut, A.: 1982, A\&A, 115, 23

Cowley, A.: 1973, PASP 85, 314

Cowley, A.P.: 1976, PASP 88, 95

Crawford, D.L.: 1975, AJ, 80, 955

ESA: 1997, The Hipparcos and Tycho Catalogues, ESA SP-1200, ESA Publications Division, Noordwijk

Fehrenbach, Ch.: 1972, A\&A 19, 427

Flower, P.J.: 1996, ApJ, 469, 355

Ginestet, N., Carquillat, J.-M., Jaschek, C., Jaschek, M.: 1997, A\&AS 123, 135

Hauck, B., Mermilliod, J.-C.: 1998, A\&AS, 129, 431

Houk, N., Smith-Moore, M.: 1988, Catalogue of two-dimensional spectral types for the HD stars, Ann Arbor, Dep. Astron, Univ. Michigan, Vol. 4

Hut, P.: 1981, A\&A, 99, 126

Malaroda, S., 1975: AJ 80, 673

Meynet, G., Mermilliod, J.-C., Maeder, A.: 1993, A\&AS, 98, 477

Moon, T.T., Dworetsky, M.M.: 1985, MNRAS, 217, 305

Moore, J.H., Paddock, G.F.: 1950, ApJ 112, 48

Nadal, R., Carquillat, J.-M., Pédoussaut, A., Ginestet, N.: 1983, A\&AS, 52, 293 (Paper I).

Nassau, J.J., MacRae, D.A.: 1955, ApJ 121, 32

Nordström, B., Stefanik, R.P., Latham, D.W., Andersen, J.: 1997, A\&AS, 126, 21

Schaller, G., Schaerer, D., Meynet, G., Maeder, A.: 1992, A\&AS, 96, 269

Schmidt-Kaler, Th.: 1982, in Landolt-Börnstein, Numerical Data and Functional Relationships in Science and Technology, K. Schaifers \& H.H. Voigt eds., New Series, Gr. VI, Vol. 2b (Springer-Verlag, Berlin), pp 1-35

Udry, S., Mayor, M., Queloz, D.: 1999, in Precise Stellar Radial Velocities, ASP Conferences Ser., 185, 367 
Wilson, R.E.: 1953, General Catalogue of Stellar Radial Velocities, Carnegie Institution Washington Publ. 601

Zahn, J.-P.: 1975, A\&A 41, 329

Zahn, J.-P.: 1977, A\&A 57, 383 
Table 4 Orbital elements of HD 54901, HD 120544 and HD 123280. In Col. 3, $T$ is the epoch of periastron passage.

\begin{tabular}{|c|c|c|c|c|c|c|c|c|c|c|}
\hline Name & $\begin{array}{c}P \\
\text { (days) }\end{array}$ & $\begin{array}{c}T \\
2400000+ \\
(J D)\end{array}$ & $\begin{array}{c}\omega \\
\text { (deg.) }\end{array}$ & $e$ & $\begin{array}{c}K_{1} \\
K_{2} \\
\left(\mathrm{~km} \cdot \mathrm{s}^{-1}\right)\end{array}$ & $\begin{array}{c}V_{0} \\
\left(\mathrm{~km} \cdot \mathrm{s}^{-1}\right)\end{array}$ & $\begin{array}{c}a_{1} \sin i \\
a_{2} \sin i \\
(\mathrm{Gm})\end{array}$ & $\begin{array}{c}M_{1} \sin ^{3} i \\
M_{2} \sin ^{3} i \\
\left(\mathrm{M}_{\odot}\right)\end{array}$ & $\begin{array}{l}f(m) \\
\left(\mathrm{M}_{\odot}\right)\end{array}$ & $\begin{array}{c}\sigma(O-C)_{1} \\
\sigma(O-C)_{2} \\
\left(\mathrm{~km}^{-1}\right)^{-1}\end{array}$ \\
\hline \multirow{3}{*}{$\begin{array}{l}\text { HD54901 } \\
\text { (Nordström } \\
\text { et al. 1997) }\end{array}$} & 41.00333 & 47709.088 & 349.3 & 0.638 & 44.46 & -9.86 & - & - & - & 0.72 \\
\hline & \pm 0.00125 & \pm 0.024 & \pm 0.5 & \pm 0.004 & \pm 0.26 & \pm 0.15 & - & - & & \\
\hline & & & & & $\begin{array}{l}58.45 \\
\pm 0.72 \\
\end{array}$ & & - & - & - & 2.36 \\
\hline \multirow{4}{*}{$\begin{array}{l}\text { HD54901 } \\
\text { (this work) }\end{array}$} & 41.00285 & 47709.105 & 349.0 & 0.629 & 44.62 & -9.82 & 19.56 & 1.245 & - & 0.83 \\
\hline & \pm 0.00030 & \pm 0.016 & \pm 0.3 & \pm 0.002 & \pm 0.16 & \pm 0.10 & \pm 0.11 & \pm 0.022 & & \\
\hline & & & & & 58.47 & & 25.64 & 0.950 & & 1.38 \\
\hline & & & & & \pm 0.27 & & \pm 0.16 & \pm 0.018 & & \\
\hline \multirow[t]{2}{*}{ HD120544 } & 89.1477 & 45025.31 & 175.3 & 0.634 & 17.57 & -30.35 & 16.65 & - & 0.0232 & 0.61 \\
\hline & \pm 0.0043 & \pm 0.23 & \pm 1.0 & \pm 0.007 & \pm 0.22 & \pm 0.12 & \pm 0.33 & - & \pm 0.0014 & \\
\hline \multirow[t]{2}{*}{ HD123280 } & 36.35588 & 45015.91 & 88.5 & 0.046 & 17.68 & 3.15 & 8.831 & - & 0.0208 & 0.38 \\
\hline & \pm 0.00060 & \pm 0.63 & \pm 6.1 & \pm 0.005 & \pm 0.09 & \pm 0.06 & \pm 0.048 & - & \pm 0.0003 & \\
\hline
\end{tabular}

Table 5 Physical parameters of the stars belonging to the binary systems derived from observational and theoretical data $\left({ }^{*}\right.$ in Col. 9: minimum value for $M_{2}$ ).

\begin{tabular}{|c|c|c|c|c|c|c|c|c|c|c|}
\hline Star & $\begin{array}{c}V \\
B-V\end{array}$ & $\begin{array}{c}v_{1} \sin i \\
v_{2} \sin i \\
\left(\mathrm{~km} \cdot \mathrm{s}^{-1}\right)\end{array}$ & (mas) & $\overline{M_{v}}$ & $\begin{array}{c}T_{\text {eff1 }} \\
T_{\text {eff2 }} \\
(\mathrm{K})\end{array}$ & $\begin{array}{l}\log g \\
(\mathrm{cgs})\end{array}$ & {$[\mathrm{Fe} / \mathrm{H}]$} & $\begin{array}{c}M_{1} \\
M_{2} \\
\left(\mathrm{M}_{\odot}\right)\end{array}$ & $\begin{array}{c}R_{1} \\
R_{2} \\
\left(\mathrm{R}_{\odot}\right)\end{array}$ & (years) \\
\hline HD5 & 7.22 & $16.3 \pm 1.0$ & 10.48 & 2.32 & $6940 \pm 75$ & 4.1 & +0.04 & $1.55 \pm 0.08$ & $1.81 \pm 0.25$ & 9.15 \\
\hline & 0.40 & & 1. & \pm 0.23 & 632 & & & 1.2 & $1.25 \pm$ & $05 /$ \\
\hline HD1 & $\begin{array}{l}6.54 \\
0.51\end{array}$ & 23.5 & $\begin{array}{l}11.11 \\
\pm 0.84\end{array}$ & $\begin{array}{c}1.77 \\
\pm 0.17\end{array}$ & $6400=$ & 4.0 & +0.09 & $\begin{array}{c}1.70 \pm 0.08 \\
0.48 \pm 0.03^{*}\end{array}$ & $3.22 \pm$ & $\begin{array}{c}9.25 \\
+0.1 /-0.1\end{array}$ \\
\hline 80 & $\begin{array}{l}8.64 \\
0.47\end{array}$ & 0.5 & $\begin{array}{c}9.80 \\
\pm 0.92\end{array}$ & $\begin{array}{c}3.60 \\
\pm 0.20\end{array}$ & $6450 \pm 90$ & 4.2 & -0.01 & $\begin{array}{c}1.30 \pm 0.05 \\
0.39 \pm 0.01^{*}\end{array}$ & $1.35 \pm 0.17$ & $\begin{array}{c}9.30 \\
+0.1 /-0.5\end{array}$ \\
\hline
\end{tabular}




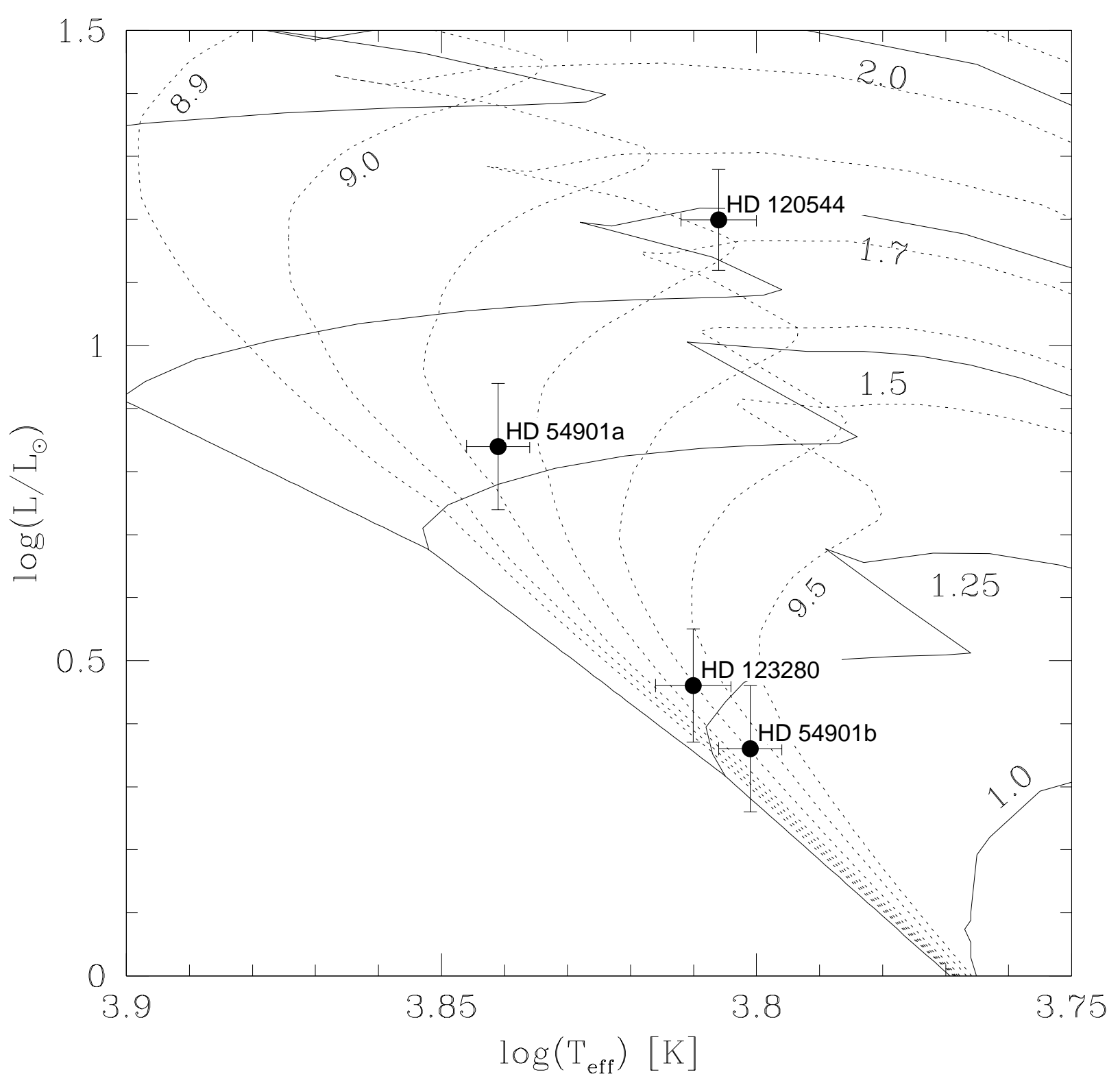

Fig. 2 Location of the components of the binary systems in the theoretical evolutionary HR diagram computed by Schaller et al. (1992) for $Z=0.02$, with the isochrones (dotted lines) given by Meynet et al. (1993), for log age[years] varying from 8.9 to 9.5 by steps of 0.1. The solid lines correspond to the evolution tracks for mass values of $1.0,1.25,1.5,1.7$ and $2.0 M_{\odot}$. 